\section{Response to a Case Report titled: Anaesthetic Management of a Patient with Left Ventricular Non-Compaction Undergoing Laparotomy}

Sir,

We read with great interest the case report titled "Anaesthetic Management of a Patient with Left Ventricular Non-Compaction Undergoing Laparotomy" by Anwar UI Huda and Tauseef Ahmed. The case report was published in special issue of September 2018 (Volume 28 No: 9). Authors have reported the successful anaesthetic management of young girl having a rare congenital cardiomyopathy called left ventricular noncompaction (LVNC) syndrome. The case was managed wonderfully giving special consideration to hemodynamic variables and goal directed fluid administration as monitored by LIDCO. We have concerns related to administration of subarachnoid block (SAB) followed by induction with general anaesthesia (GA). Though there is no consensus about selection of particular anaesthetic technique ${ }^{1}$, but the authors in that case are justified with patient's baseline health status and well-preserved cardiac functions. Besides, they have facilities of routine follow-ups and advance hemodynamic monitoring, which is recommended for the safe conduct of anaesthesia. ${ }^{2}$ However, for health care setups where facilities are limited and follow-up mechanisms are vague, we have to be very careful in terms of following similar anaesthetic regime. These patients rely on baseline sympathetic tone and alteration in after load to maintain their cardiac output (CO). Administration of $S A B$ followed by GA may be deleterious in terms of massive sympethectomy, vasodilation, and reduction in preload. ${ }^{3}$ This may lead to life-threatening drop in $\mathrm{CO}$, myocardial hypo-perfusion, and increase risk of arrhythmias. The message needs to be clear that this option is limited only for selected number of patients having the availability of tertiary care facilities.

Moreover, the prediction of risk and selection of particular anaesthetic plan should be based on status of ejection fraction 4 in patients with cardiomyopathy.

\section{REFERENCES}

1. Smit-Fun V, Buhre WF. The patient with chronic heart failure undergoing surgery. Curr Opin Anaesthesiol 2016; 29:391-6.

2. Kapoor PM, Magoon R, Rawat RS, Mehta Y, Taneia S, Ravi R, et al. Goal-directed therapy improves the outcome of high-risk cardiac patients undergoing off-pumpcoronary artery bypass. Ann Card Anaesth 2017; 20:83-9.
3. Van Diepen S, Bakal JA, McAlister FA, Ezekowitz JA. Mortality and readmission and of patients with heart failure, atrial fibrillation, or coronary artery disease undergoing non-cardiac surgery: An analysis of 38,047 patients. Circulation 2011; 124 : 289-96.

4. Upshaw J, Kiernan M. Preoperative cardiacs risk assessment for non-cardiac surgery in patients with heart failure. Curr Heart Fail Rep 2013; 10:147-56.

Faraz Shafiq and Muhammad Irfan Ul Haq

Department of Anaesthesiology, The Aga Khan University, Karachi, Pakistan

Correspondence: Dr. Faraz Shafiq, Department of Anaesthesiology, The Aga Khan University, Karachi, Pakistan E-mail: faraz.shafiq@aku.edu

Received: October 08, 2018; Accepted: November 08, 2018

\section{Author's Reply:}

We are thankful for the comments about our case report. It was pointed out in the letter that anaesthetic choice needs to be individualised in every case. GA along with $S A B$ is well established technique. As we mentioned in our case report that the patient had well preserved cardiac function and the main consideration in this case was risk of perioperative arrhythmias; that is why our priority in this case was providing adequate anaesthesia and analgesia and avoiding sympathetic stimulation that could lead to perioperative arrythmias. We completely agree that sympathectomy and a drop in systemic peripheral resistance can be disastrous in vulnerable patients. This was the reason, we had used LIDCO rapid in this case to keep a very close eye on all the haemodynamic variables. We used a good dose of intrathecal diamorphine, rather, as this helps in better pain control in postoperative period.

I agree that SAB will not be a good option in patients who have significant cardiac dysfunction. Additionally, irrespective of the anaesthesia technique used intraoperatively, these patients will need facilities like high dependency unit in postoperative period because of higher risk of arrhythmias. Further, the key aspect of this case was to identify the risks preoperatively, communicating with the medical team for optimisation and utilising the available resources appropriately in our District General Hospital.

Anwar ul Huda

Department of Anaesthesia, Southport and Ormskirk NHS

Hospital, UK

Correspondence: Dr. Anwar ul Huda, Department of Anaesthesia, Southport and Ormskirk NHS Hospital, UK

E-mail: hudaanwar90@yahoo.com ........ 